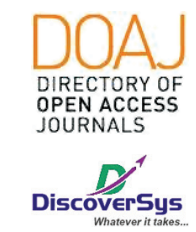

Published by DiscoverSys

\section{Periodontitis as early detection of diabetes mellitus: a literature review}

\author{
Ade Indah Pratiwi*
}

Postgraduate student, Department of Physiology, Universitas Udayana, Bali, Indonesia
${ }^{*}$ Correspondece to: Ade Indah Pratiwi; Postgraduate student, Department of Physiology, Universitas Udayana, Bali, Indonesia; gecindahpratiwi@gmail.com

Received: 2019-09-17 Accepted: 2020-01-04 Published: 2020-03-03

\title{
ABSTRACT
}

Background: Diabetes mellitus (DM) is a growing health problem and its prevalence is dramatically increasing, particularly in Indonesia. It can affect every organ in the body, and one of the oral manifestations is periodontitis characterized by the loss of tissue attachment. This review aims to identify further the periodontitis as early detection of diabetes mellitus

Methods: A review of relevant literature was performed to elaborate on periodontitis involvement in diabetes mellitus. A total of 25 qualified published literature of all years were collected from several electronic database and manual search and included in this review.

Results: On average, patients with periodontitis showed higher glycated haemoglobin (HbA1c) plasma levels compared with individuals without periodontitis. Diabetes and periodontitis are complex chronic diseases with an established bidirectional relationship. There is long-established evidence that hyperglycemia in diabetes is associated with adverse periodontal outcomes. Here, we show that periodontitis is an early sign of diabetes mellitus and may, therefore, serve as a valuable risk indicator. A dentist who got patient with periodontitis is a suitable location for screening for diabetes by a simple finger stick and validated HbA1c dry spot analysis.

Conclusion: Literature from a different type of study concluded that periodontitis is one of the oral manifestations that could be used as early detection in diabetes mellitus

Keywords: diabetes mellitus, periodontitis, periodontal disease

Cite this Article: Pratiwi, A.I. 2020. Periodontitis as early detection of diabetes mellitus: a literature review. Intisari Sains Medis 11(1): 108-111. DOl: 10.15562/ism.v11i1.623

\section{INTRODUCTION}

Diabetes mellitus (DM), particularly type-2 DM, is a growing health problem and its prevalence is dramatically increasing. The prevalence of diabetes mellitus in Indonesia increased annually based on Riskesdas in 2013. ${ }^{1}$ Indonesia occupies 4th place with the number of diabetics the biggest after India, China and the United States. In 2030, Indonesia is expected to have people with DM as many as 21.3 million people. ${ }^{1}$ In addition, type-2 DM is the most type often found in dental practice, about $90 \%$ of all DM patients in Indonesia. ${ }^{2}$ Epidemiological data confirm that diabetes is a significant risk factor for periodontitis; susceptibility to periodontitis is increased by approximately threefold in people with diabetes. This is a clear relationship between the degree of hyper-glycaemia and periodontitis.

\section{An overview of periodontitis}

Periodontitis is one of periodontal disease as the most common diseases known to humankind. Periodontal disease includes two significant entities, gingivitis and periodontitis. Gingivitis is inflammation of periodontal disease characterized by reversible gingiva destruction, but present periodontitis destruction of the tooth-supporting structure may with irreversible and also may tooth loss. ${ }^{3}$ Tissue destruction in periodontitis results in a breakdown of the collagen fibres of the periodontal ligament, resulting in the formation of a periodontal pocket between the gingiva and the tooth.

Periodontitis is a slowly progressing disease, but the tissue destruction that occurs is mostly irreversible. In the early stages, the condition is typically asymptomatic; it is not usually painful, and many patients are unaware until the condition has progressed enough to result in tooth mobility. ${ }^{3}$ The pockets deepen as a result of the further destruction of fibres of the periodontal ligament and the resorption of the alveolar bone that occurs in parallel with the progressing attachment loss. 'Pocket' is not evident on simple visual inspection, and assessment using a periodontal probe is essential. Advanced periodontitis is characterized by gingival erythema and oedema, gingival bleeding, gingival recession, tooth mobility, drifting of teeth, suppuration from periodontal pockets, and tooth loss. ${ }^{4}$

Moderate periodontitis is the most conditions affecting $40-60 \%$ of adults with highly prevalent but primarily hidden in chronic inflammation. ${ }^{5}$ Severe periodontitis that threatens tooth retention affecting $10-15 \%$ of adults in the most population studied. ${ }^{5}$ Prevalence of periodontitis increased with age up to the point that $70.1 \%$ of adults $\geq 65$ years old were affected by periodontal disease. ${ }^{6}$ Men exhibit 
worse periodontal status than women $[(56.4 \%$ vs $38.4 \%$ ), as well as those with limited education $(66.9 \%)$ and income $(65.4 \%)]$. The risk factor of periodontitis increased with smoking habits. It harms many aspects of daily living and quality of life, affecting confidence, social interaction and food choices. ${ }^{7}$

People with diabetes have other risk factors of periodontitis such as condition associated with compromised immune responses (e.g. HIV), nutritional defect, osteoporosis, medication because of drug-induced gingival overgrowth, genetic factor and local factor. ${ }^{8}$ The incidence of periodontitis increases and can be more severe in patients who have DM. This is not always directly proportional to plaque and calculus because periodontitis is also influenced by environmental factors including the condition of the teeth that are crowded, metabolic, genetic, and age. Many studies show a reciprocal relationship between periodontitis and DM by comparing between periodontitis in the DM group and periodontitis in the Non-DM group. ${ }^{9}$

\section{Aetiology of periodontitis}

Plaque accumulation on teeth produces gingivitis, but the degree of inflammation and destruction of the alveolar bone that supports teeth depend on the host susceptibility. ${ }^{10}$ The inflammatory response is characterized by secretion of host-derived mediators of inflammation and tissue breakdown. Interleukin- $1 \beta$, interleukin- 6 , prostaglandin $\mathrm{E} 2$ (PGE2), tumour necrosis factor alpha (TNF- $\alpha$ ), receptor activator of nuclear factor $\kappa \mathrm{B}$ ligand (RANKL), and matrix metalloproteinases (MMP8, MMP-9, and MMP-13) are the most commonly implicated mediators of inflammation. ${ }^{11}$

Hyperglycemia increases the concentration of glucose in the saliva and the gingival crevicular fluid. This leads to the proliferation of bacteria in the oral cavity. Hyperglycemia itself also has an indirect adverse effect, stimulating the immune system's cells to release inflammatory cytokines. ${ }^{12}$ Elevated levels of proinflammatory mediators in the periodontal pockets result in osteoclastic destruction. Diabetic microangiopathy, impaired immune response, and lower resistance to infections contribute to the development of periodontitis in poorly controlled diabetics. ${ }^{12}$

The continuous exposure of collagen fibres in the supporting periodontal ligaments to aldose sugars induces their nonenzymatic glycation and oxidation. This glycation leads to changes in the physical properties of these molecules, reducing collagen solubility and increasing the degradation of connective tissues. This results in accelerated deterioration of both connective tissue and bone. ${ }^{12}$

\section{Effect of Periodontitis on Glycemia}

Longitudinal studies have demonstrated that severe periodontitis is associated with poorly controlled glycemia, higher HbA1c levels and development of systemic diabetic complications. ${ }^{13}$ It also has been reported that periodontitis is associated with a slight elevation of $\mathrm{HbAlc}$ in nondiabetic subjects (periodontitis may potentially increase the incidence of diabetes), although a clear-cut association could not be established. ${ }^{14}$ Studies assumed that periodontal infection might impair glycemic control by increasing insulin tissue resistance. ${ }^{15}$

Diabetes and periodontitis are complex chronic diseases with an established bidirectional relationship. There is long-established evidence that hyperglycemia in diabetes is associated with adverse periodontal outcomes. ${ }^{16}$ Type 2 diabetes is preceded by an ill-defined period of systemic inflammation, leading to reduced pancreatic beta cell function, increased beta cell apoptosis, and insulin resistance. Periodontitis may stimulate systemic inflammation, the oral cavity providing a means for the entry of periodontal organisms and their virulence factors into the circulation.

Inflammation-mediated oxidative-stress pathways and advanced glycation end products (AGEs)-receptor for AGEs (RAGE) interactions provide plausible links in the periodontitis to diabetes direction. The periodontium is a highly vascular tissue and any inflammation there may serve as a gateway to the systemic circulation for bacterial products and locally produced inflammatory mediators. ${ }^{12}$ The infectious challenge contributes to insulin resistance by modification of insulin receptor substrate- 1 by serine phosphorylation, altered adipocyte function with increased production of free fatty acids, and diminution of endothelial nitric oxide production. The process may further alter pancreatic $\beta$-cell function, either acting directly or through stimulation of free fatty acid production. ${ }^{12}$

\section{Correlation between diabetes and periodontitis}

Diabetes affects every organ in the body, and one of the oral manifestations is periodontitis characterized by the loss of tissue attachment The disease characterized by an increased susceptibility to infection, poor wound healing, and increased morbidity and mortality associated with disease progression. Diabetes also recognized as an important risk factor for more severe and progressive periodontitis, infection or lesions resulting in the destruction of tissues and supporting bone that forms the attachment around the tooth. ${ }^{17}$ 
The level of glycemic control is of key importance in determining increased risk. For example, in the US National Health and Nutrition Examination Survey (NHANES) III, adults with an HbAlc level of $>9 \%$ had a significantly higher prevalence of severe periodontitis than those without diabetes (OR 2.90; 95\% CI 1.40, 6.03) after controlling for age, ethnicity, education, sex and smoking. ${ }^{18}$ The majority of research has focused on type 2 diabetes mellitus as a risk factor for periodontitis, probably because both diseases have historically tended to develop in patients in their 40s and 50s. ${ }^{18}$ However, type 1 diabetes mellitus also increases the risk of periodontitis, and all patients with diabetes (including children and young adults) should be considered to be at increased risk of periodontitis. ${ }^{19}$ One early study identified that around $10 \%$ of children $(<18$ years) with type 1 diabetes mellitus had increased attachment loss and bone loss compared with controls, despite comparable plaque scores. ${ }^{19}$

Dentists have long been aware of the importance of a diagnosis of diabetes in their patients, and various oral conditions are associated with diabetes, including xerostomia and candidal infections as well as periodontitis. In the early 1990s periodontitis was sometimes referred to as the sixth complication of diabetes', and in 2003 the ADA acknowledged that periodontal disease is often found in people with diabetes. ${ }^{16,20}$

\section{Periodontal treatment is associated with improved glycemic control}

Several meta-analyses have confirmed that effective periodontal therapy can result in reduced HbAlc. The first reported on ten interventional studies with a combined population of 456 patients; the authors identified a weighted mean reduction in $\mathrm{HbAlc}$ of $0.66 \%$ as a result of periodontal therapy. ${ }^{21}$ In 2008 , a meta-analysis of nine studies involving 485 patients reported a substantial reduction in $\mathrm{HbAlc}$ of $0.46 \%$ following periodontal treatment. ${ }^{22}$ In 2010 , a meta-analysis of five studies involving 371 patients also reported a significant weighted mean reduction in $\mathrm{HbA} 1 \mathrm{c}$ of $0.40 \%$ over a follow-up period of 3-9 months after periodontal therapy. ${ }^{23}$

Taken collectively, the evidence supports the notion that improvements in metabolic control can be anticipated following effective treatment of periodontitis (although there are few studies available, and some studies lack power). ${ }^{24}$ The mechanisms by which this occurs are not yet clear but probably relate to reduced systemic inflammation (e.g. reduced serum levels of mediators such as TNF- $\alpha$ and IL-6) following the treatment and resolution of periodontal inflammation. More extensive randomized trials are warranted to investigate this further. These observations are important because reductions in $\mathrm{HbAlc}$ are associated with a reduced risk of diabetes complications. For example, each $1 \%$ reduction in $\mathrm{HbAlc}$ or poor glycemic control in type-2 diabetes mellitus has been associated with reductions in risk of $21 \%$ for any endpoint related to diabetes, $21 \%$ for deaths related to diabetes, $14 \%$ for myocardial infarction and 37\% for microvascular complications. ${ }^{24,25}$

\section{CONCLUSION}

Diabetes and periodontitis are the two most commonly encountered chronic disease. Considering how closely interlinked these are, it is imperative to look for one when encountering each other. The early identification of subjects at high risk for diabetes mellitus or with undiagnosed diabetes mellitus is crucial to implement measures that may prevent or delay progression from pre-diabetes to overt diabetes mellitus and reduce the incidence of chronic complications. Severe periodontitis is more frequently found in diabetic subjects with high HbAlc levels and systemic diabetic complications; however, the influence of periodontal treatment on HbAlc is not that well established. There is long-established evidence that hyperglycemia in diabetes is associated with adverse periodontal outcomes. Here, we show that periodontitis is an early sign of diabetes mellitus and may, therefore, serve as a valuable risk indicator. A dentist who got patient with periodontitis is a suitable location for screening for diabetes by a simple finger stick and validated HbAlc dry spot analysis.

\section{CONFLICT OF INTEREST}

There is no competing interest regarding the manuscript.

\section{FUNDING}

None

\section{AUTHOR CONTRIBUTION}

Ade Indah Pratiwi is responsible for the study from beginning the conceptual framework, searching literature, analyze, and synthesis the literature review in narrative form.

\section{REFERENCES}

1. Idris H, Hasyim H, Utama F. Analysis of Diabetes Mellitus Determinants in Indonesia: A Study from the Indonesian Basic Health Research 2013. Acta Med Indones. 2017;49(4):291-298.

2. Mihardja L, Soetrisno U, Soegondo S. Prevalence and clinical profile of diabetes mellitus in productive aged urban Indonesians. J Diabetes Investig. 2014;5(5):507-12. 
3. Listgarten MA, Schifter CC, Laster L. 3-year longitudinal study of the periodontal status of an adult population with gingivitis. J Clin Periodontol 1985;12:225-238

4. Preshaw PM, Alba AL, Herrera D, Jepsen S, Konstantinidis A, Makrilakis $\mathrm{K}$, et al. Periodontitis and diabetes: a two-way relationship. Diabetologia. 2012;55(1):21-31.

5. Fox CH. New considerations in the prevalence of periodontal disease. Curr Opin Dent. 1992 Mar;2:5-11.

6. Eke PI, Dye BA, Wei L, Thornton-Evans GO, Genco RJ; CDC Periodontal Disease Surveillance workgroup, et al. Prevalence of periodontitis in adults in the United States: 2009 and 2010. J Dent Res. 2012;91(10):914-20.

7. O`Dowd LK' , Durham J, McCracken GI, Preshaw PM. Patients' experiences of the impact of periodontal disease. J Clin Periodontol. 2010;37(4):334-9.

8. Pihlstrom BL, Michalowicz BS, Johnson NW. Periodontal diseases. Lancet. 2005;366(9499):1809-20.

9. Grover HS, Luthra S. Molecular mechanisms involved in the bidirectional relationship between diabetes mellitus and periodontal disease. J Indian Soc of Periodontol. 2013;17(3):292 - 301

10. Trombelli L, Tatakis DN, Scapoli C, Bottega S, Orlandini E, Tosi M. Modulation of clinical expression of plaque-induced gingivitis. II. Identification of "high-responder" and "low-responder" subjects. J Clin Periodontol 2004;31(4):239-252.

11. Preshaw PM, Taylor JJ. How has research into cytokine interactions and their role in driving immune responses impacted our understanding of periodontitis? J Clin Periodontol 2011;38(Suppl 11):60-84.

12. Sharma M, Jindal R, Siddiqui MA, Wangnoo SK. Diabetes and Periodontitis: A medical perspective. 2016;8(1):3-7

13. Chapple IL, Genco R; working group 2 of the joint EFP/ AAP workshop. Diabetes and periodontal diseases: consensus report of the Joint EFP/AAP Workshop on Periodontitis and Systemic Diseases. J Periodontol 2013;84(4 Suppl): S106-S112.

14. Wolff RE, Wolff LF, Michalowicz BS. A pilot study of glycosylated hemoglobin levels in periodontitis cases and healthy controls. J Periodontol 2009;80(7):1057-1061

15. Bascones-Martínez A, Muñoz-Corcuera M, BasconesIlundain J. Diabetes and periodontitis: A bidirectional relationship. Med Clin (Barc) 2015;145(1):31-35
16. Expert Committee on the Diagnosis and Classification of Diabetes Mellitus. Report of the expert committee on the diagnosis and classification of diabetes mellitus. Diabetes Care 2003;26(Suppl 1):S5-20

17. Southerland JH, Taylor GW, Offenbacher S. Diabetes and Periodontal Infection: Making the Connection. 2005;23(4):171-178

18. Tsai C, Hayes C, Taylor GW. Glycemic control of type 2 diabetes and severe periodontal disease in the US adult population. Community Dent Oral Epidemiol. 2002;30(3):182-92.

19. Cianciola LJ, Park BH, Bruck E, Mosovich L, Genco RJ. Prevalence of periodontal disease in insulin-dependent mellitus (juvenile diabetes). J Am Dent Assoc. 1982;104(5):653-60.

20. Löe H. Periodontal disease. The sixth complication of diabetes mellitus. Diabetes Care. 1993;16(1):329-34.

21. Janket SJ, Wightman A, Baird AE, Van Dyke TE, Jones JA. Does periodontal treatment improve glycemic control in diabetic patients? A meta-analysis of intervention studies. J Dent Res. 2005;84(12):1154-9.

22. Darré L, Vergnes JN, Gourdy P, Sixou M. Efficacy of periodontal treatment on glycaemic control in diabetic patients: a meta-analysis of interventional studies. Diabetes Metab. 2008;34(5):497-506.

23. Teeuw WJ, Gerdes VE, Loos BG. Effect of periodontal treatment on glycemic control of diabetic patients: a systematic review and meta-analysis. Diabetes Care. 2010;33(2):421-7.

24. Al-Zahrani MS, Borawski EA, Bissada NF. Increased physical activity reduces prevalence of periodontitis. J Dent. 2005;33(9):703-10.

25. Artha IMJR, Bhargah A, Dharmawan NK, Pande UW, Triyana KA, Mahariski PA, et al. High level of individual lipid profile and lipid ratio as a predictive marker of poor glycemic control in type-2 diabetes mellitus. Vascular Health and Risk Management. 2019;15:149-157

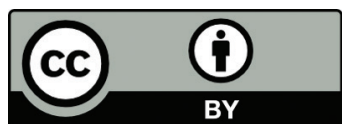

This work is licensed under a Creative Commons Attribution 